\title{
Free Recall Learning of Hierarchically Organised Lists by Adults with Asperger's Syndrome: Additional Evidence for Diminished Relational Processing
}

\author{
Dermot M. Bowler · Sebastian B. Gaigg • \\ John M. Gardiner
}

Published online: 21 November 2008

(C) The Author(s) 2008. This article is published with open access at Springerlink.com

\begin{abstract}
The Task Support Hypothesis (TSH, Bowler et al. Neuropsychologia 35:65-70 1997) states that individuals with autism spectrum disorder (ASD) show better memory when test procedures provide support for retrieval. The present study aimed to see whether this principle also applied at encoding. Twenty participants with high-functioning ASD and 20 matched comparison participants studied arrays of 112 words over four trials. Words were arranged either under hierarchically embedded category headings (e.g. Instruments-String-Plucked-Violin) or randomly. Both groups showed similar overall recall and better recall for the hierarchically organised words. However, the ASD participants made less use of information about relations between words and more use of item-specific information in their recall, confirming earlier reports of relational difficulties in this population.
\end{abstract}

Keywords Asperger's syndrome - Memory · Free recall · Categories $\cdot$ Relational encoding $\cdot$ Learning

There is now a considerable literature that shows that individuals with all types of autism spectrum disorders (ASDs) at times experience difficulties in using semantic aspects of learned material to aid their free recall of that

An earlier report of the work was presented at the International Meeting for Autism Research, Boston, May 2005.

D. M. Bowler $(\bowtie) \cdot$ S. B. Gaigg · J. M. Gardiner

Autism Research Group, Department of Psychology, City

University, Northampton Square, London, UK

e-mail: d.m.bowler@city.ac.uk

J. M. Gardiner

University of Sussex, Brighton, UK material. Studies of children with classic Kanner-type ASD have shown diminished use of semantic relatedness in free recall (Hermelin and O'Connor 1967; O'Connor and Hermelin 1967; Tager-Flusberg 1991), and similar difficulties have been demonstrated in adults with highfunctioning ASD or Asperger's syndrome (Bowler et al. 1997; Smith et al. 2007, but see Leekam and Lopez 2003 for contradictory findings). Although the early observations led Hermelin and O'Connor (1970) to conclude that memory difficulties in autism resulted from a failure to encode stimuli meaningfully, more recent evidence suggests that some aspects of semantic relatedness are encoded by individuals with ASD. Children and adults with high and low-functioning ASD show undiminished cued recall when superordinate category cues are used (Boucher and Warrington 1976; Minshew et al. 1992) and adults with high-functioning ASD are susceptible to semantically induced memory interference effects (Bowler et al. 2006). In addition such individuals are subject to the DRM memory illusion effect (Deese 1959; Roediger and McDermott 1995), in which a list of strong associates of a non-studied word is learned. At later test, most studies have shown that the non-studied associate is recalled or recognised, indicating awareness of the associative structure of the studied list (Beversdorf et al. 2000; Beversdorf et al. 2007; Bowler et al. 2000), although Beversdorf et al. (2000) have reported enhanced discrimination of the nonstudied item.

The question raised by the pattern of results just described centres on why semantic or associative relatedness should be encoded by individuals with ASD, yet not be available to enhance performance on tasks such as free recall. One possible explanation centres on the Task Support Hypothesis (TSH), developed by Gardiner, Bowler and colleagues (Bowler et al. 1997, 2004). The TSH attempts to 
account for the patterning of memory performance seen in ASD across different kinds of memory test. People with ASD tend to show diminished performance on memory tasks when unsupported test procedures, such as free recall are used but when supported test procedures, such as recognition or cued recall are used, there is less evidence of diminished performance (see Bowler and Gaigg 2008 for a review). This pattern also holds for incidentally-encoded material and has been shown to account for inconsistent findings regarding source memory in ASD (Bowler et al. 2004). Most important for the current context, however, is the finding that individuals with ASD seem to be able to draw on semantic relationships to facilitate their performance on tests of recognition but not on tests of free recall (Bowler et al. 2008a).

Although there is strong evidence in favour of the TSH at the level of retrieval, the question remains about whether the hypothesis can be extended to encoding. Gaigg et al. (2008) compared the effects on free recall of sorting words into categories versus rating their pleasantness and found that these orienting tasks improved free recall by adults with ASD and typical development by a similar margin. Similarly, Gaigg and Bowler (2008) recently found that individuals with ASD exhibited typically enhanced memory for semantically related versus unrelated words that were encoded through a pleasantness rating task. By contrast, no improvements in recall were found to result from instructions in which participants were told how to rehearse items from categorised lists (Smith et al. 2007). On the basis of these findings, it appears that individuals with ASD need to be given more than just instructions about the categorised nature of the studied material in order to profit from support at encoding. It would seem that they need to engage actively with the study material. The somewhat inconsistent findings on memory for categorised information described above may be resolved with reference to the psychological processes involved in learning semantically related groups of words. Memory phenomena that are produced by inter-item associations (such as memory illusions) involve a less complex level of awareness than those that stem from organisation of items into hierarchical categories (see Bowler and Gaigg 2008; Gaigg et al. 2008). On this distinction, differential performance across experiments can be accounted for in terms of the relational complexity (Halford 1993) of the tasks. Individuals with ASD have a tendency to engage in item specific rather than relational processing of studied items in memory tasks and this processing bias seems sufficient to allow them to make use of inter-item associations of a non-hierarchical kind. Thus when they encode a series of related items, they tend to process inter-item relations on the basis of already stored knowledge about each item but not on the basis of more complex frameworks that also make use of how inter-item relations themselves relate to higher order categorical groupings.

It is also possible that the single trial procedures that have characterised most of the published investigations to date may be insufficient to make ASD participants aware of semantic relations in the way that they might do if they were required to study the same list several times over. Up to now, there have been few attempts to study the evolution of free recall of the same list of items over successive learning trials. Two studies have utilised the California Verbal Learning test with HFA adults (Minshew and Goldstein 1993) and adolescents (Bennetto et al. 1996). This test requires participants to learn a list of 16 words from four categories over five consecutive trials. Both investigators found diminished recall on later trials and Minshew and Goldstein also found diminished clustering of items by category at recall in the ASD group. Bowler et al. (2008b) adapted a procedure developed by Tulving (1962) in which participants with and without HFA were asked to free recall a list of 16 unrelated words over a series of 16 trials with the words presented in a different order on each trial. Bowler et al.'s results showed among other things marginally diminished recall by the HFA participants compared to a typical comparison group, thus demonstrating that the HFA group continue to experience difficulty in free recall learning.

Given that the free recall by people with HFA of both categorised and uncategorised lists improves more slowly over trials than that of comparison participants, the present investigation was designed to test the hypothesis that free recall learning of categorised sets of items would be enhanced by providing support at encoding. This was done by explicitly highlighting the hierarchically categorised structure of the studied material. We employed a procedure developed by Bower et al. (1969) in which participants were asked to study the same set of 112 words presented for $3 \mathrm{~min}$ on four consecutive trials. The words consisted of category labels (e.g. minerals, vegetables), sub-category labels (e.g. metals, further sub-divided into rare, common, alloys) and exemplars (e.g. platinum, iron, amalgam). The set of words to be studied was presented either in a layout that emphasised the hierarchical, categorical structure of the set, or in the same layout as the hierarchical list but with words distributed randomly, irrespective of whether they were category, sub-category or exemplar labels. At the end of each 3-min study period, participants were asked to free recall as many of the items as they could. Bower et al. (1969) found that recall rates improved over trials and that organisation at study increased participants' overall recall rates. Our aim was to see if provision of explicit clues to the hierarchical structure of the studied material would improve the free recall of individuals with ASD over trials, and whether the rate of such improvement was similar to 
that of typical comparison participants. In view of the documented differential operation of item-specific over relational processing in ASD, we also proposed to examine these processes quantitatively using Category Access (CAcc) and Items per Category (IPC) measures that are generally agreed to reflect relational and item-specific processing respectively (Burns and Brown 2000; Hunt and Seta 1984). CAcc is defined as the number of categories from which participants recall at least one item, whilst IPC is defined as the average number of items participants recall from each of these categories. On the basis of existing research (e.g. Gaigg et al. 2008), we would predict higher IPC and diminished CAcc in the ASD sample. There is also the possibility that the strong hierarchical clues provided in the present study would yield an increase in CAcc in the ASD group. Our question is whether the free recall performance of individuals with ASD would improve to the same extent as that of matched typical individuals in both structured and unstructured presentation.

\section{Method}

\section{Participants}

Twenty individuals with ASD (4 female, 16 male) and 20 typical individuals (4 female, 16 male) participated in the current experiment. ASD and comparison participants were individually matched to within 7 points of verbal IQ as measured by the third edition of the Wechsler Adult Intelligence Scale (WAIS-III ${ }^{\mathrm{UK}}$; The Psychological Corporation, 2000) and groups did not differ in terms of their chronological age, performance IQ or full-scale IQ. The relevant data for these group characteristics are set out in Table 1. Participants with ASD were diagnosed according to conventional criteria and a review of available medical records and/or assessment with the Autism Diagnostic Observation Schedule (ADOS; Lord et al. 1989) confirmed that all met DSM-IV-TR (American Psychiatric Association 2000) criteria for an Autism Spectrum Disorder.

Table 1 Average age and IQ scores for the ASD and typical comparison group

\begin{tabular}{|c|c|c|c|c|}
\hline \multirow[t]{2}{*}{ Measure } & \multicolumn{2}{|c|}{$\operatorname{ASD}(N=20)$} & \multicolumn{2}{|c|}{ Comparison $(N=20)$} \\
\hline & $M$ & $S D$ & $M$ & $S D$ \\
\hline Age (years) & 33.0 & 13.1 & 30.4 & 10.0 \\
\hline $\mathrm{VIQ}^{\mathrm{a}}$ & 107.8 & 15.5 & 107.4 & 14.7 \\
\hline $\mathrm{PIQ}^{\mathrm{b}}$ & 107.8 & 16.8 & 105.1 & 12.5 \\
\hline $\mathrm{FIQ}^{\mathrm{c}}$ & 108.6 & 16.8 & 104.6 & 12.4 \\
\hline
\end{tabular}

${ }^{\text {a }}$ Verbal IQ (WAIS-R ${ }^{\text {UK }}$ or WAIS-III ${ }^{\mathrm{UK}}$ )

${ }^{b}$ Performance IQ (WAIS-R ${ }^{\mathrm{UK}}$ or WAIS-III ${ }^{\mathrm{UK}}$ )

${ }^{c}$ Full-Scale IQ (WAIS-R ${ }^{\mathrm{UK}}$ or WAIS-III ${ }^{\mathrm{UK}}$ )

Participants comprising the typical comparison group were recruited through local newspaper advertisements and included in the current study only if they were free of psychotropic medication and did not report any family history of neuropathology or psychiatric illness. All participants gave their informed consent to take part in the experiment and all were remunerated standard University fees for their participation.

\section{Materials and Design}

Following Bower et al. (1969), the experimental materials for the current study consisted of two sets (Set A and Set B) of four conceptual hierarchies, which were generated by representing the hierarchical nature of conceptual categories in the form of hierarchical trees. An example of one of these trees is shown in Fig. 1. As illustrated in this example, each hierarchy consisted of 4 levels of category specificity. Level 1 always consisted of a single superordinate category label, which included minerals, instruments, vegetables and insects for Set A and buildings, plants, arts and industries for Set B. Levels 2 and 3 represented category labels of increasing specificity and Level 4 consisted of exemplars for each of the Level 3 categories. Although the precise structure of the different hierarchical trees varied somewhat, each hierarchy included five sets of category exemplars at Level 4. All but one of the categories consisted of 28 words in total with the remaining category (i.e. Insects) consisting of 27 words. Thus in total Set A consisted of 111 words, whilst Set $\mathrm{B}$ consisted of 112 words.

(a)

\begin{tabular}{lllll}
\multicolumn{2}{c}{ String } & & \multicolumn{2}{c}{ Wind } \\
Plucked & Bowed & Percussion & Wood & Brass \\
Guitar & Cello & Xylophone & Bassoon & Saxophone \\
Zither & Violin & Triangle & Flute & Trombone \\
Lute & Fiddle & Tambourine & Oboe & Trumpet \\
Banjo & Bass & Drum & Clarinet & Tuba
\end{tabular}

Cockroach

(b)

Brass

Sprouts

Bowed

Wasp

Woodlouse

Pewter

Brass
Fig. 1 Examples of conceptually organised (a) and randomly organised (b) hierarchical presentation of words

\section{Gold}

$\begin{array}{llll}\text { Masonry } & \text { Trumpet } & \text { Tambourine } & \text { Pest } \\ \text { Copper } & \text { Fiddle } & \text { Zither } & \text { Chicory } \\ \text { Marrow } & \text { Greens } & \text { Slate } & \text { Bee } \\ \text { Seedpod } & \text { Bass } & \text { Flea } & \text { Parsnip } \\ \text { Granite } & \text { Spinach } & \text { Vegetables } & \text { Oboe }\end{array}$


The principal aim of the current experiment was to evaluate the extent to which structured presentation at study could enhance participants' recall of displays of hierarchically-organised words. For this purpose participants were presented with one set of conceptually organised hierarchies and one set of randomly constructed hierarchies. For the conceptually organised display, the four hierarchies within a set were displayed in four quadrants of a PowerPoint slide (Microsoft Office, 2003) with the conceptual relations of items within each hierarchy left intact (i.e. as shown in Fig. 1). For the random display, words were presented in an identical hierarchical format but the positions of words were randomised across the four hierarchies and across the four levels of the hierarchical trees (obvious conceptual relationships among proximal words were avoided, see Fig. 1). Words were presented in white Arial font on a black background and in order to facilitate visibility of the words, the materials were projected onto a plain white wall approximately 2 metres in front of the participant. The resulting displays measured approximately $1.7 \times 0.9 \mathrm{~m}\left(46^{\circ} \times 25^{\circ}\right.$ of visual angle $)$ with each hierarchy occupying an area of about $70 \times 40 \mathrm{~cm}\left(20^{\circ} \times 11^{\circ}\right.$ of visual angle $)$. The resulting font size subtended approximately $2.5^{\circ}$ of visual angle.

\section{Procedure}

Participants were tested individually in a sound attenuated laboratory dimly lit by a fluorescent desk lamp. For pilot purposes we measured participants' eye-gaze throughout the experiment, which required them to wear a headmounted eye-tracker (RK-726 PCI Pupil/Corneal reflection tracking system; ISCAN Inc. 2001) consisting of a baseball cap equipped with 2 unobtrusive cameras. Following the set-up and calibration procedures for the eye-tracker, participants were involved in conversation for approximately 5-10 min in order for them to adjust to the equipment.

During the memory experiment participants were asked to learn both sets of hierarchies over four successive trials each. The first set of hierarchies to be memorised always consisted of the randomised display of either Set A or Set B whilst the second set of hierarchies consisted of the conceptually organised display of the other set. Sets A and B were counterbalanced across the randomised and conceptually organised display conditions but the order of testing (i.e. randomized before conceptually organised) was fixed in order to avoid alerting participants to the hierarchical nature of stimuli for the randomized displays.

Participants were informed that during the experiment they would be asked to try to remember a set of about 100 words over four successive trials. They were told that on each trial the words would be projected onto the wall all at once for $3 \mathrm{~min}$ and that after each trial they would be required to say out loud as many words as possible. It was explained that following a break the entire procedure would be repeated with a different set of words. No mention was made about the categorical nature of the stimuli or the hierarchical format of the displays. Once participants understood what they were asked to do the hierarchical displays were presented and participants' oral recall was recorded for later analysis.

\section{Results}

The veridical recall data (proportions of total words studied) for the ASD and comparison group over the four trials of the random and conceptually organized display conditions are set out in Fig. 2. A 2 (Group; ASD vs. Comparison) $\times 2$ (Display; Random vs. Organized) $\times 4$ (Trial) mixed ANOVA of these data revealed main effects for Display $(F(1,38)=78.09, \quad p<.001)$ and Trial $(F(3,36)=147.40, p<.001$; Greenhouse-Geisser correction) indicating that performance was better for the organized $(M=0.44 ; S D=0.21)$ as compared to the random display $(M=0.26 ; S D=0.13)$ and that all participants' performance improved over trials. The results were furthermore characterised by a significant interaction between the Display and Trial factors $(F(3,36)=19.80$, $p<.001$; Greenhouse-Geisser correction). Post hoc analyses revealed that this interaction was due to a significantly bigger increase in items recalled over successive trials during the organised $(M=0.11 ; S D=.06)$ as compared to the random display condition $(M=0.07 ; S D=0.04)$ $(t=5.39, \quad$ df $=39, \quad P<.001)$. Finally, the analysis revealed a marginal effect of Group $(F(1,38)=3.75$, $P=.06)$ with poorer performance in the ASD $(M=0.30$; $S D=0.18)$ as compared to the comparison group $(M=.39 ; S D=.12)$ (Fig. 2).

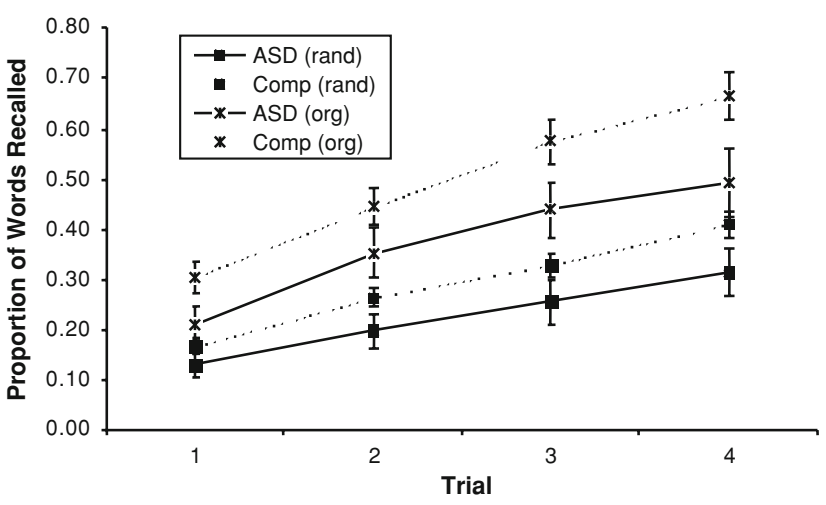

Fig. 2 Proportion of words recalled from the random (rand) and conceptually organized (org) displays over trials as a function of group 
As indicated in our introduction, we have recently suggested that the memory difficulties characterising individuals with ASD stem from relatively specific problems with relational but not item-specific memory processes (Gaigg et al. 2008). In order to test this hypothesis in relation to the current experiment we computed the CAcc and IPC measures, which are widely thought to reflect relational and item-specific memory processes respectively (e.g. Hunt and Seta 1984; Burns and Brown 2000). In the current paradigm these indices of relational and item-specific processing can be computed at each of the three levels of category specificity (the fourth level of each hierarchy represents category exemplars and not another sub-category). Since the pattern of significant effects and interactions was similar across the three levels and because ceiling effects were most marked for measures derived from Level 1 and 2 of the hierarchies, we only present results from a detailed analysis of CAcc and IPC scores derived from the third level of the hierarchies. Nevertheless, the CAcc data from the first trial of Level 1 are worth describing briefly. Ninety per cent of typical participants recalled at least one item from each of the four hierarchies when these were presented randomly and $70 \%$ when they were presented hierarchically. The respective percentages for the ASD participants are $60 \%$ and $30 \%$. $^{1}$ The number of individuals who reported at least one member of a Level 1 hierarchy on Trial 1 was significantly different between groups both for organised presentations $(t=6.24, \mathrm{df}=1$, $p<.05)$ and for random presentations $(t=4.68, \mathrm{df}=1$, $p<.05)$. These data parallel those from earlier single-trial studies of relational encoding in ASD (e.g. Gaigg et al. 2008).

At the third level of hierarchies participants could recall a maximum of 20 categories ( 5 for each of the 4 hierarchies) and a maximum of 5 words per category (the level 3 label plus 4 exemplars). As is the convention, we report the respective CAcc and IPC data as proportions of these maxima. Table 2 sets out the Level 3 CAcc and IPC data as a function of group, display type and trial. A 2 (Group; ASD vs. Comparison) $\times 2$ (Display; Random vs. Organized) $\times 4$ (Trial) mixed ANOVA of the CAcc measure revealed main effects for Display $(F(1,38)=4.79$, $p<.05)$, Trial $(F(3,36)=167.80, p<.001$, GreenhouseGeisser correction) and Group $(F(1,38)=9.32, p<.01)$ but no significant interactions between the factors. These results reflect superior category access for the organised versus the random display condition and superior category access on successive trials. The main effect of group

\footnotetext{
1 This observation appears counter-intuitive, as we would expect higher recall of Level 1 items under the structured display. However the difference is in the same direction for both groups of participants, suggesting that it results from some strategic aspect of participants' recall of the highest elements of the hierarchy.
}

reflects the fact that ASD participants recalled overall fewer categories than comparison participants, reflecting the findings from the first trial of Level 1 described above. The lack of any interactions involving the group factor (all $F$ s $<0.5)$ suggests that the meaningfulness of the conceptual organisation of stimuli as well as their repeated presentation facilitated category access in both groups by a similar margin. As for the IPC data, an equivalent analysis also revealed main effects for Display $(F(1,38)=162.33$, $p<.001)$ and Trial $(F(3,36)=47.08, p<.001$, Greenhouse-Geisser correction) again reflecting superior performance on the organised versus the random display condition and on successive trials. Unlike the results from the analysis of the category access measure, however, the group factor yielded no main effect $(F(3,36)=1.44$, ns).

Finally, to rule out the possibility that the findings reported above might have resulted from between-group differences in intrusions or repetitions, we assessed whether groups may have differed in terms of falsely recalling words that they had not actually seen. Overall, such intrusions were relatively rare with the ASD group producing an average of $1.95(S D=2.26)$ new words over the four random display trials and $4.00(S D=3.97)$ new words over the conceptually organised trials. The respective numbers of intrusions for the typical comparison group were $2.40(S D=2.62)$ and $3.70(S . D .=3.85)$. Thus, whilst the number of intrusions was significantly higher for the conceptually organised than the random display condition $(F(1,38)=10.79, p<.01)$, groups did not differ in this respect $(F(1,38)=0.01, n s)$. Equally we found no differences between groups in terms of how many times

Table 2 Mean and standard errors of the Category Access (CA) and Item Per Category (IPC) measures for the ASD and Typical comparison group as a function of display and trial

\begin{tabular}{|c|c|c|c|c|}
\hline & \multicolumn{4}{|l|}{ Trial } \\
\hline & 1 & 2 & 3 & 4 \\
\hline \multicolumn{5}{|c|}{ Random display } \\
\hline \multicolumn{5}{|c|}{ Asperger } \\
\hline CA & $.42(.05)$ & $.56(.05)$ & $.64(.05)$ & $.71(.03)$ \\
\hline IPC & $.33(.02)$ & $.36(.03)$ & $.40(.04)$ & $.46(.04)$ \\
\hline \multicolumn{5}{|c|}{ Comparison } \\
\hline $\mathrm{CA}$ & $.54(.03)$ & $.73(.02)$ & $.79(.03)$ & $.85(.02)$ \\
\hline IPC & $.34(.01)$ & $.39(.02)$ & $.45(.02)$ & $.53(.02)$ \\
\hline \multicolumn{5}{|c|}{ Conceptually organised display } \\
\hline \multicolumn{5}{|c|}{ Asperger } \\
\hline $\mathrm{CA}$ & $.43(.05)$ & $.61(.05)$ & $.70(.05)$ & $.73(.06)$ \\
\hline IPC & $.54(.03)$ & $.60(.05)$ & $.65(.05)$ & $.68(.05)$ \\
\hline \multicolumn{5}{|c|}{ Comparison } \\
\hline $\mathrm{CA}$ & $.61(.05)$ & $.76(.04)$ & $.87(.03)$ & $.92(.03)$ \\
\hline IPC & $.55(.03)$ & $.64(.04)$ & $.72(.04)$ & $.79(.44)$ \\
\hline
\end{tabular}


participants repeated certain words during any given trial $(F(1,38)=0.31, \mathrm{~ns})$. The ASD group repeated on average $8.65(S D=8.96)$ words per trial during the random display condition and $6.70(S D=5.26)$ during the conceptually organised display condition whilst the respective values for the typical comparison group were $8.85(S D=6.76)$ and $8.85(S D=8.95)$. The analysis of these data also revealed no significant differences between the random and conceptually organised conditions $(F(1,38)=0.65, \mathrm{~ns})$.

\section{Discussion}

Our results confirm our hypotheses that individuals with ASD would show lower free recall and diminished CAcc scores when compared to typical individuals. These findings replicate and extend earlier work on multi-trial free recall learning in individuals with ASD (Bowler et al. 2008b; Minshew and Goldstein 1993). The absence of differential effects of display organisation on recall for the two groups supports our conjecture, based on the TSH, that the ASD and comparison participants would benefit to an equal extent from the hierarchical presentation of some of the lists at study. However, the observation of diminished CAcc in the ASD group together with a similar rate of increase in these scores over trials for the two groups shows that the ASD participants, although less likely to organise their recall around the categorical structure of the studied items, nevertheless appear to benefit from the presence of such structure over trials. In this respect, the findings replicate those of Gaigg et al. (2008), who showed that individuals with ASD, although more likely to engage in item-specific processing of studied material, benefited from an orientation task that emphasised categorical, relational encoding.

The findings discussed so far extend the application of the TSH from retrieval to encoding, but a closer inspection of the findings shows that overall recall improvement over trials may have resulted from the operation of different processes in the two groups of participants. In particular, the absence of a group difference in the IPC measure, coupled with its presence in the CAcc measure, suggests that the ASD participants are relying more heavily on itemspecific rather than relational processing to aid their free recall. This finding is in line with other studies that show diminished relational processing in this group (Bowler et al. 2008a; Gaigg et al. 2008). It might also help to explain why studies that simply provided information at encoding about the categorical structure of the study list (Bowler et al. 2006) or on how to use this information when rehearsing studied material (Smith et al. 2007) did not show enhanced recall.
The support provided by the present findings to the hypothesis that memory difficulties in ASD are the result of difficulties in the relational processing of material further strengthens the view that individuals with ASD have difficulties with the processing of relationally complex information (Bowler and Gaigg 2008). Moreover, relational processing underlies a range of memory phenomena such as source memory, episodic remembering and enhanced recall of categorised lists, all of which have been shown to be diminished in individuals with ASD (Bowler et al. 1997, 2000, 2004). The fact that these difficulties are more in evidence when unsupported test procedures are employed leads us to conclude that even when clues to the relational nature of the studied material are provided at study, as in the present investigation and in that of Gaigg et al. (2008), recall difficulties still remain evident in this group, suggesting that although task support can enhance performance, it may do so by promoting patterns of processing that are particular to individuals with ASD and which continue to yield diminished performance in that group.

When considered alongside the results of earlier studies (e.g. Smith et al. 2007), the findings reported here have important implications for the design of programmes of instruction for use with people with ASD. Care should be taken to ensure active involvement at study when elements of the learned material have to be organised into higherorder structures. Similarly, in circumstances where the learned material is to be utilised, attention needs to be focused on the provision of adequate clues to the relevant higher-order aspects of the information.

Acknowledgement The work reported in this paper was supported by a project grant from the Wellcome Trust to the first and second authors.

Open Access This article is distributed under the terms of the Creative Commons Attribution Noncommercial License which permits any noncommercial use, distribution, and reproduction in any medium, provided the original author(s) and source are credited.

\section{References}

American Psychiatric Association. (2000). Diagnostic and statistical manual of mental disorders, 4th Edition-Text Revision. Washington, DC: APA.

Bennetto, L., Pennington, B. F., \& Rogers, S. J. (1996). Intact and impaired memory function in autism. Child Development, 67, 1816-1835. doi:10.2307/1131734.

Beversdorf, D. Q., Smith, B. W., Crucian, G. P., Anderson, J. M., Keillor, J. M., Barrett, A. M., et al. (2000). Increased discrimination of "false memories" in autism spectrum disorder. Proceedings of the National Academy of Sciences of the United States of America, 97, 8734-8737. 
Beversdorf, D. Q., Narayanan, A., Hillier, A., \& Hughes, J. D. (2007). Network model of decreased context utilization in autism spectrum disorder. Journal of Autism and Developmental Disorders, 37, 1040-1048. doi:10.1007/s10803-006-0242-7.

Boucher, J., \& Warrington, E. K. (1976). Memory deficits in early infantile autism: Some similarities to the amnesic syndrome. The British Journal of Psychology, 67, 73-87.

Bower, G. A., Clark, M. C., Lesgold, A. M., \& Winzenz, D. (1969). Hierarchical retrieval schemes in recall of categorized word lists. Journal of Verbal Learning and Verbal Behavior, 8, 323-343. doi:10.1016/S0022-5371(69)80124-6.

Bowler, D. M., \& Gaigg, S. B. (2008). Memory in ASD: Emerging themes and future prospects. In J. Boucher \& D. M. Bowler (Eds.), Memory in Autism: Theory and evidence. Cambridge: Cambridge University Press.

Bowler, D. M., Gaigg, S. B., \& Gardiner, J. M. (2006). Multiple list learning in high-functioning ASD: Limits to the Task Support Hypothesis. Poster presented at the International Meeting for Autism Research, Montreal, May 2006.

Bowler, D. M., Gaigg, S. B., \& Gardiner, J. M. (2008a). Effects of related and unrelated context on recall and recognition by adults with high-functioning autism spectrum disorder. Neuropsychologia, 46, 993-999. doi:10.1016/j.neuropsychologia.2007.12.004.

Bowler, D. M., Gaigg, S. B., \& Gardiner, J. M. (2008b). Subjective organisation in the free recall of adults with Asperger's syndrome. Journal of Autism and Developmental Disorders, 38, 104-113. doi:10.1007/s10803-007-0366-4.

Bowler, D. M., Gardiner, J. M., \& Berthollier, N. (2004). Source memory in Asperger's syndrome. Journal of Autism and Developmental Disorders, 34, 533-542. doi:10.1007/s10803004-2548-7.

Bowler, D. M., Gardiner, J. M., Grice, S., \& Saavalainen, P. (2000). Memory illusions: False recall and recognition in high functioning adults with autism. Journal of Abnormal Psychology, 109, 663-672. doi:10.1037/0021-843X.109.4.663.

Bowler, D. M., Matthews, N. J., \& Gardiner, J. M. (1997). Asperger's syndrome and memory: Similarity to autism but not amnesia. Neuropsychologia, 35, 65-70. doi:10.1016/S0028-3932(96) 00054-1.

Burns, D. J., \& Brown, C. A. (2000). The category access measure of relational processing. Journal of Experimental Psychology. Learning, Memory, and Cognition, 26, 1057-1062. doi: 10.1037/0278-7393.26.4.1057.

Deese, J. (1959). On the prediction of occurrence of particular verbal intrusions in immediate recall. Journal of Experimental Psychology, 58, 17-22. doi:10.1037/h0046671.

Gaigg, S. B., \& Bowler, D. M. (2008). Free recall and forgetting of emotionally arousing words in autism spectrum disorder. $\mathrm{Neu}$ ropsychologia, 46, 2336-2343. doi:10.1016/j.neuropsychologia. 2008.03.008.
Gaigg, S. B., Bowler, D. M., \& Gardiner, J. M. (2008). Free recall in autism spectrum disorder: The role of relational and itemspecific encoding. Neuropsychologia, 46, 986-992.

Halford, G. S. (1993). Children's understanding: The development of mental models. Hillsdale, NJ: L. Erlbaum.

Hermelin, B., \& O'Connor, N. (1967). Remembering of words by psychotic and subnormal children. The British Journal of Psychology, 58, 213-218.

Hermelin, B., \& O'Connor, N. (1970). Psychological experiments with autistic children. Oxford: Pergamon Press.

Hunt, R. R., \& Seta, C. E. (1984). Category size effects in recall: The role of relational and item-specific information. Journal of Experimental Psychology. Learning, Memory, and Cognition, 10, 454-464. doi:10.1037/0278-7393.10.3.454.

Leekam, S., \& Lopez, B. (2003). Do children with autism fail to process information in context? Journal of Child Psychology and Psychiatry and Allied Disciplines, 44, 285-300. doi:10.1111/ 1469-7610.00121.

Lord, C., Rutter, M., Goode, S., Heemsbergen, J., Jordan, J., Mawhood, L., et al. (1989). Autism Diagnostic Observation Schedule: A standardised observation of communicative and social behavior. Journal of Autism and Developmental Disorders, 19, 185-212. doi:10.1007/BF02211841.

Minshew, N. J., \& Goldstein, G. (1993). Is autism an amnesic disorder? Evidence from the California Verbal Learning Test. Neuropsychology, 7, 209-216. doi:10.1037/0894-4105.7.2.209.

Minshew, N., Goldstein, G., Muenz, L. R., \& Payton, J. (1992). Neuropsychological functioning in nonmentally retarded autistic individuals. Journal of Clinical and Experimental Neuropsychology, 14, 749-761.

O'Connor, N., \& Hermelin, B. (1967). The selective visual attention of autistic children. Journal of Child Psychology and Psychiatry and Allied Disciplines, 8, 167-179. doi:10.1111/j.14697610.1967.tb02192.x.

Roediger, H. L., III, \& McDermott, K. B. (1995). Creating false memories: Remembering words not presented in lists. Journal of Experimental Psychology. Learning, Memory, and Cognition, 21, 803-814. doi:10.1037/0278-7393.21.4.803.

Smith, B. J., Gardiner, J. M., \& Bowler, D. M. (2007). Deficits in free recall persist in Asperger's syndrome despite training in the use of list-appropriate learning strategies. Journal of Autism and Developmental Disorders, 37, 445-454.

Tager-Flusberg, H. (1991). Semantic processing in the free recall of autistic children: Further evidence for a cognitive deficit. British Journal of Developmental Psychology, 9, 417-430.

Tulving, E. (1962). Subjective organisation in the free recall of "unrelated" words. Psychological Review, 69, 344-354. 